\title{
Chronic Urticaria Associated With Occult Papillary Thyroid Carcinoma and Dramatic Improvement After Total Thyroidectomy: A Retrospective Study From Turkey
}

\author{
Özkaya E, Kılıç Sayar S, Mahmudov A \\ Department of Dermatology and Venereology, Istanbul Faculty \\ of Medicine, Istanbul University, Istanbul \\ J Investig Allergol Clin Immunol 2019; Vol. 29(6): 465-467 \\ doi: 10.18176/jiaci.0435
}

Key words: Thyroid carcinoma. Papillary. Chronic urticaria. Thyroid ultrasonography. Thyroidectomy.

Palabras clave: Carcinoma de tiroides. Papilar. Urticaria crónica. Ultrasonografía de tiroides. Tiroidectomía.

Chronic urticaria (CU) is characterized by recurrent hives lasting more than 6 weeks, either alone or in combination with angioedema, and may persist for months to years [1]. It is classified as spontaneous or inducible and can be further classified into subtypes such as dermographic, cholinergic, aquagenic, solar, and cold-, heat-, or pressure-induced. The various triggers proposed include foods, drugs, infections, autoimmune comorbidities (including autoimmune thyroid disease), and cancer [1]. Recent reports indicated associations with hematological malignancies such as leukemia, lymphoma, and myeloma, as well as with carcinoma of the lung, bladder, testis, ovary, and thyroid gland, in particular papillary thyroid carcinoma. The dramatic recovery from urticaria after total thyroidectomy in cases of thyroid carcinoma suggests a causal relationship (Table) [2-5]. The aim of this study was to analyze the frequency of thyroid carcinoma and outcome of treatment among patients with chronic urticaria.

The study population comprised 359 patients who were diagnosed with chronic urticaria (both spontaneous and inducible). The diagnosis was based on clinical findings and the results of provocation testing performed at our dermatology-allergy clinic between 2010 and 2018. All patients had undergone a routine thyroid work-up including thyroid-stimulating hormone (TSH) level, thyroid function tests (free thyroxine, free triiodothyronine), thyroid antibodies (antithyroglobulin and antithyroperoxidase [TPO]), and thyroid ultrasound, which was the key diagnostic tool for suspecting thyroid carcinoma in otherwise asymptomatic patients. Fine-needle aspiration biopsy was performed if thyroid ultrasound raised the suspicion of a nodule measuring $\geq 10 \mathrm{~mm}$ in diameter. Patients with malignant or suspicious cytology findings underwent total thyroidectomy. Patients diagnosed with thyroid carcinoma according to histopathology findings were evaluated in regard to the duration of the disease, age, gender, and treatment outcomes using the weekly urticaria activity score (UAS7).

Focal and systemic infections, food and drug allergy, and urticarial vasculitis were ruled out. Elevated levels of thyroid 


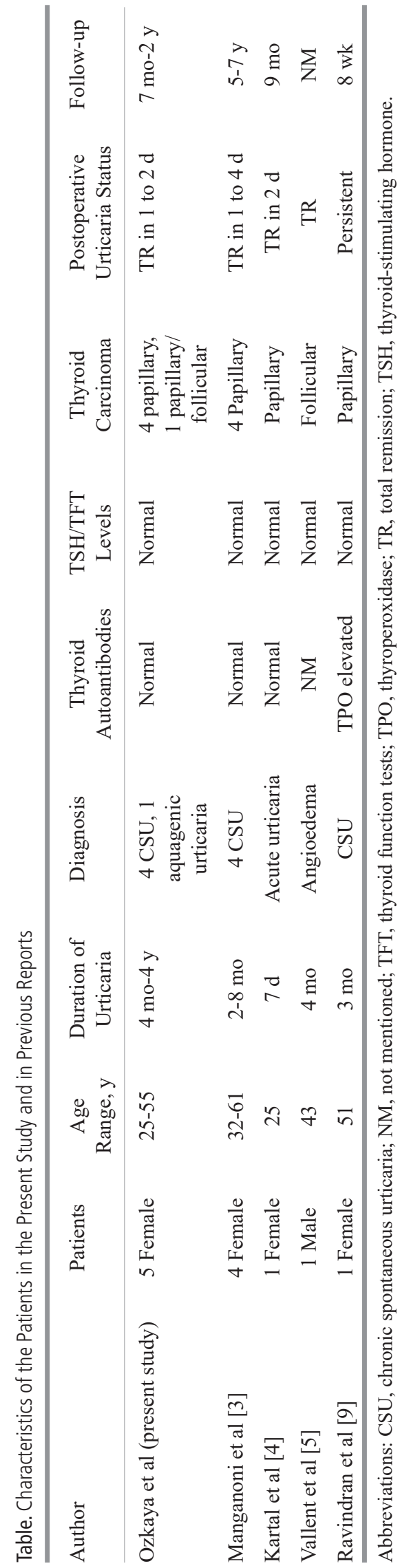

autoantibodies were detected in 118 patients, and 21 patients had abnormal results in their thyroid function tests or TSH level. A total of 30 patients had previously known thyroid disease (14 had elevated levels of thyroid autoantibodies, and 7 had abnormalities in their thyroid function and/or TSH tests), such as Hashimoto disease $(\mathrm{n}=23)$, benign multinodular goiter $(n=3)$, papillary thyroid carcinoma $(n=2)$, hypothyroidism of unknown etiology $(n=1)$, and hyperthyroidism $(n=1)$. In the 2 patients with papillary thyroid carcinoma, thyroidectomy had been performed 8-9 years before the onset of urticaria.

Suspected nodules measuring $\geq 10 \mathrm{~mm}$ in diameter were identified by thyroid ultrasound in 27 patients, who subsequently underwent fine-needle aspiration biopsies. Cytology revealed malignancy in 5 patients, suspicion in 2 patients, and benign lesions in the remaining patients. All 5 patients with malignancy underwent total thyroidectomy. Histopathology showed occult papillary thyroid carcinoma in 4 patients and occult papillary/follicular thyroid carcinoma in 1 patient (Table). Patients were exclusively female. The mean age of onset of urticaria was 29.8 years (range, 7-59 years). No patients had a previous history of thyroid disease or other autoimmune disorder. There were no clinically palpable thyroid nodules. Thyroid function tests and serum levels of TSH and thyroid autoantibodies were within normal limits. Four of the patients had chronic spontaneous urticaria, and 1 patient had chronic inducible urticaria, ie, aquagenic urticaria. The latter was described in detail in a recent report [2]. No other triggers for urticaria were found, and patients were taking regular antihistamine therapy. The median time between the onset of urticaria and diagnosis of thyroid carcinoma was 6 months (range, 4 months- 4 years). The prevalence of thyroid carcinoma-associated chronic urticaria was $1.4 \%$ (5/359).

Total remission of urticaria was achieved in 4 patients within 1-2 days following total thyroidectomy. The UAS7 decreased from a mean score of 32.2 (range, 21-42) to zero in all patients. Urticaria did not recur in these patients after a median follow-up period of 47 months. In 1 patient, urticaria had remitted by $80 \%$ the day after the operation. Urticaria resolved completely within 2 weeks and did not recur during a 2-year follow-up.

Two patients with a suspicious cytology result also underwent total thyroidectomy. Histopathology revealed benign lesions in both cases. One of these patients, who had intermittent chronic urticaria, reported that she was free of attacks after surgery and for a further 5 years while on therapy with thyroid hormones. The other patient was lost to follow-up after the surgical procedure.

The incidence of thyroid carcinoma is increasing worldwide, probably owing to easier detection of occult cases. Papillary thyroid carcinoma is the most frequent type of thyroid carcinoma and more commonly affects females [6]. It also seems to be the main type of thyroid carcinoma associated with urticaria. Recent reports showed a significant association between papillary thyroid carcinoma and chronic spontaneous urticaria in 4 patients [3] and acute urticaria in 1 patient [4]; of the 5 patients, 4 were female (Table). Follicular thyroid carcinoma was associated with isolated angioedema in 1 patient [5]. In a further 2 cases, angioedema-like lesions resulted from superior vena cava obstruction secondary to thyroid carcinoma $[7,8]$. The improvement in urticaria in a 
very short period following total thyroidectomy-both in the present study and in previously published reports (Table) — was striking. This suggests a causal relationship between urticaria and thyroid carcinoma, rather than a coincidence. All patients had occult thyroid carcinoma that could only be suspected by thyroid ultrasound. Consistent with our findings, thyroid autoantibodies, TSH, and thyroid function tests were within normal limits in all of these previously reported patients except in 1 case with elevated levels of TPO antibodies [9]. Urticaria persisted only in this case [9]. The patient's TPO antibody titer returned to normal values after thyroidectomy, and the authors suggested that her urticaria could have been complicated by insufficiently suppressed TSH after surgery [9].

Interestingly, in our study, 1 of the patients who underwent total thyroidectomy owing to a suspicious cytology finding that proved to be benign reported that she had had no further attacks of urticaria after surgery or during a 5-year follow-up.

The real prevalence of thyroid carcinoma in patients with chronic urticaria remains to be determined. A population-based cohort study from Taiwan reported an increased risk of thyroid cancer in patients with chronic urticaria, although no further details were provided [10].

Our study shows that routine thyroid ultrasound alone might serve as a life-saving diagnostic tool in patients with chronic urticaria who were "asymptomatic" for thyroid carcinoma and prevent these patients from expensive and unnecessarily long therapy for chronic urticaria. As the most striking finding, chronic urticaria resolved dramatically in all of the 5 patients with thyroid carcinoma within 1-2 days following total thyroidectomy. Therefore, we would suggest ultrasound-based screening of the thyroid gland in all patients with chronic urticaria, regardless of the results of TSH/thyroid function tests and serum thyroid antibody titers.

\section{Funding}

The authors declare that no funding was received for the present study.

\section{Conflicts of Interest}

The authors declare that they have no conflicts of interest.

\section{References}

1. Chiu HY, Muo CH, Sung FC. Associations of chronic urticaria with atopic and autoimmune comorbidities: a nationwide population-based study. Int J Dermatol. 2018;57:822-9.

2. Ozkaya E, Elinç-Aslan MS, Yazici S. Aquagenic urticaria and syncope associated with occult papillary thyroid carcinoma and improvement after total thyroidectomy. Arch Dermatol. 2011;147:1461-2.

3. Manganoni AM, Tucci G, Venturini M, Farisoglio C, Baronchelli C, Calzavara Pinton PG. Chronic urticaria associated with thyroid carcinoma: report of 4 cases. J Investig Allergol Clin Immunol. 2007;17:192-5.

4. Kartal O, Abdullah B, Ramazan E, Mustafa G, Zafer C, Osman S. Acute urticaria associated with thyroid papillary carcinoma: a case report. Ann Dermatol. 2012;24:453-4.
5. Vallent K, Fehér J, Somogyi A, Joós A. Angioneurotic edema and hypocomplementemia in patients with thyroid cancer. Acta Chir Acad Sci Hung. 1982;23:163-71.

6. Gonzalez-Gonzalez R, Bologna-Molina R, Carreon-Burciaga RG, Gómezpalacio-Gastelum M, Molina-Frechero N, Salazar-Rodríguez S. Papillary thyroid carcinoma: differential diagnosis and prognostic values of its different variants: review of the literature. ISRN Oncol. 2011;2011:915925. doi: 10.5402/2011/915925

7. Eastman JJ, Kim AS. A patient with idiopathic angioedema presenting with superior vena cava syndrome and lymphedema. Ann Allergy Asthma Immunol. 2017;118:257-8.

8. O'Donnell BF, Foulds IS. Thyroid carcinoma presenting as angio-oedema. Br J Dermatol. 1993;128:588-9.

9. Ravindran S, Melvani V, Tobin M. Chronic urticaria in the presence of papillary thyroid cancer. Ann Allergy Asthma Immunol. 2016;117 Supplement, S53.

10. Chen YJ, Wu CY, Shen JL, Chen TT, Chang YT. Cancer risk in patients with chronic urticaria: a population-based cohort study. Arch Dermatol. 2012;148:103-8

- Manuscript received April 30, 2019; accepted for publication July 22, 2019.

Sıla Kılıç Sayar

Istanbul Üniversitesi, Istanbul Tıp Fakültesi Deri ve Zührevi Hastalıklar Anabilim Dalı 34093 Çapa-Istanbul, Turkey E-mail: s_kilic@windowslive.com 